\title{
ON A THEOREM OF DESKINS
}

\author{
IRENE ZIMMERMANN
}

(Communicated by Warren J. Wong)

\begin{abstract}
In this note we present a solvability criterion for finite groups. We show that a finite group $G$ is solvable if in every maximal subgroup chain of length 3 of $G$ at least one term is a submodular subgroup of $G$. This generalizes an earlier result of Deskins.
\end{abstract}

In [2] Deskins showed that a finite group is solvable if the number of subnormal terms in every maximal subgroup chain of the group is sufficiently large. More precisely, let

$$
\left(\mathscr{C}_{n}\right): G=G_{0}>G_{1}>\cdots>G_{n}
$$

be a maximal chain of length $n$ of the group $G$, i.e., $G_{i}$ is a maximal subgroup of $G_{i-1}$ for $i=1, \ldots, n$. If $s\left(\mathscr{C}_{n}\right)$ denotes the number of those $G_{i} \neq G$ which are subnormal subgroups of $G$, then $n / s\left(\mathscr{C}_{n}\right)=v\left(\mathscr{C}_{n}\right)$ is called the (subnormal) variance of the chain $\left(\mathscr{C}_{n}\right)$, where $v\left(\mathscr{C}_{n}\right)=n$ in case none of the $G_{i}$ is subnormal in $G$. The variance $v(G)$ of the group $G$ is the maximum of all $v\left(\mathscr{C}_{n}\right)$ for all maximal chains of arbitrary length in $G$. Deskins obtained the following result:

Theorem (Deskins). The finite group $G$ is solvable if

(i) $v(G)<4$

or

(ii) $v(G)<5$ and $3 \nmid|G|$.

By a result of Thompson (cf. [4, p. 188]), $2^{6}$ divides the order of a finite non-solvable group provided that 3 is not a divisor of the group order. Using this, Asaad proved in [1]:

Theorem (Asaad). Let $G$ be a finite group such that $(|G|, 3)=1$. If $v(G)<8$, then $G$ is solvable.

The purpose of this note is to establish a solvability criterion for finite groups that generalizes the variance condition of Deskins. We show that it is not necessary to study maximal chains of arbitrary length. Actually, the solvability

Received by the editors September 21, 1988 and, in revised form, March 13, 1989.

1980 Mathematics Subject Classification (1985 Revision). Primary 20D10, 20 E1 5. 
of a finite group $G$ with $v(G)<4$ or $v(G)<8$ and $(|G|, 3)=1$ arises from the fact that in every maximal chain of $G$ a subnormal subgroup appears within the first 3, resp. 7 steps. Therefore we can restrict our attention to maximal chains of length 3, resp. 7. Moreover it is enough to require that these short maximal chains contain at least one submodular subgroup (instead of a subnormal subgroup). The definition of a submodular subgroup was introduced in [6].

Definition. A subgroup $T$ of a group $G$ is called submodular in $G$, if there exists a finite series

$$
T=T_{1}<T_{2}<\cdots<T_{s}<T_{s+1}=G
$$

such that $T_{i}$ is modular in $T_{i+1}$ for all $i=1, \ldots, s$.

Recall that a subgroup $M$ of a group $G$ is called modular in $G$ if $M$ is a modular element in the subgroup lattice of $G$. Since every normal subgroup is modular, it is clear that submodularity is a generalization of both modularity and subnormality. For an investigation of submodular subgroups in finite groups see [6].

A finite group containing a proper non-trivial submodular subgroup is not simple: If $G$ is a simple group, then $<1>$ and $G$ are the only modular elements in the lattice of subgroups of $G$. This is a consequence of a result due to Schmidt ([5, Lemma 1]):

Lemma (Schmidt). Let $M$ be a maximal modular subgroup of the finite group $G$. Then either $M$ is a maximal normal subgroup of $G$ or $G / M_{G}$ is a nonabelian group of order $p q, p$ and $q$ prime numbers.

Definition. A finite group $G$ is called an $\operatorname{sm}(\leq i)$-group if in every maximal chain

$$
\left(\mathscr{C}_{i}\right): G=G_{0}>G_{1}>\cdots>G_{i}
$$

of length $i$ at least one term $G_{j} \neq G, j \in\{1, \ldots, i\}$, is submodular in $G$.

If $k>0$, then every $\operatorname{sm}(\leq i)$-group is an $\operatorname{sm}(\leq i+k)$-group; clearly, $G$ is an $\operatorname{sm}(\leq i)$-group if $G$ does not contain maximal chains of length $i$. Consider a finite group $G$ with $v(G)<i$ and let

$$
\left(\mathscr{C}_{i}\right): G=G_{0}>G_{1}>\cdots>G_{i}
$$

be an arbitrary maximal chain of length $i$ in $G$. Since $i / s\left(\mathscr{C}_{i}\right) \leq v(G)<i$ at least two terms in the chain $\left(\mathscr{C}_{i}\right)$ must be subnormal in $G$. Omitting the last term $G_{i}$ in $\left(\mathscr{C}_{i}\right)$, we obtain a maximal chain of length $i-1$ in which at least one subnormal subgroup of $G$ appears. Therefore the following is immediate:

Every finite group $G$ with $v(G)<i$ is an $\operatorname{sm}(\leq i-1)$-group.

We now state our main result, which in particular implies the theorems of Deskins and Asaad. 


\section{Theorem.}

(i) A finite $\operatorname{sm}(\leq 3)$-group is solvable.

(ii) A finite $\operatorname{sm}(\leq 7)$-group $G$ is solvable if $(|G|, 3)=1$.

If $T$ is a submodular subgroup of a group $G$ and $T<U<G$, then $T$ is submodular in $U$. Also $T N / N$ is submodular in $G / N$ for every $N \triangleleft G$. From these observations the next lemma is obvious:

Lemma. Let $G$ be a finite $\operatorname{sm}(\leq i)$-group.

(i) Every maximal non-modular subgroup of $G$ is an $\operatorname{sm}(\leq i-1)$-group.

(ii) If $N \triangleleft G$, then $G / N$ is an $\operatorname{sm}(\leq i)$-group.

Proof of the theorem. Let $G$ provide a minimal counterexample to the assertion, i.e., $G$ is a non-solvable $\operatorname{sm}(\leq 3)$-group. First observe that $G$ cannot be simple. Otherwise, $G$ does not contain a proper non-trivial submodular subgroup and therefore every 3-maximal subgroup of $G$ is trivial. (A subgroup is called $i$ maximal if it occurs as the $i$ th term in a maximal chain of $G$.) This implies that every proper subgroup of $G$ is solvable. But a minimal non-solvable group always contains 3-maximal non-trivial subgroups (cf. [3, p. 190]).

It follows from the lemma that every non-modular maximal subgroup as well as every homomorphic image of $G$ is an $\operatorname{sm}(\leq 3)$-group.

Since a maximal modular subgroup of a non-solvable group contains a nontrivial normal subgroup, the following assumptions are justified:

(1) $G$ possesses a unique minimal normal subgroup $N$ which is nonabelian.

(2) $N$ is contained in every modular maximal subgroup of $G$.

Now let $U$ be a non-modular maximal subgroup of $G$. By the lemma, $U$ is solvable and hence $U_{G}=1$. Let $U>U_{1}>U_{2}$ be any maximal chain of length 2 in $U$. Then $U_{1}$ or $U_{2}$ must be submodular subgroups of $G$. Since $U_{G}=1$, no non-trivial subgroup of $U$ is subnormal in $G$ ([2, Lemma 1]). It follows that $U_{1}$ or $U_{2}$ is abelian of square free exponent ([6, Proposition 2]), i.e., every proper subgroup of $U_{1}$ is submodular in $G$. Thus:

(3) For $i \geq 2$ every $i$-maximal subgroup of a non-modular maximal subgroup of $G$ is submodular in $G$.

Let $N=N_{1} \times \cdots \times N_{r}$ with isomorphic simple groups $N_{i}$. Take a subgroup $P$ of prime order $p$ in $N_{1}$ and let $P \leq S \in \operatorname{Syl}_{p}(N)$. By the Frattini argument

$$
G=N_{G}(S) N
$$

which implies, in view of (2), that $N_{G}(S)$ is contained in a maximal nonmodular subgroup $V$ of $G$. We have

$$
P \leq S \leq N_{N}(S)=N_{G}(S) \cap N<N_{G}(S) \leq V .
$$

If $P$ is not a maximal subgroup of $V$, then $P$ is submodular in $G$ by (3). But this is impossible since the non-abelian simple group $N_{1}$ does not contain 
proper non-trivial submodular subgroups. Hence $P$ is maximal in $V$ and therefore

$$
P=S=N_{N}(S)
$$

By a theorem of Burnside (cf. [3, p. 419, 2.6 Hauptsatz]) $N$ contains a normal $p$-complement. This contradiction proves (i).

For the proof of (ii) we choose again a minimal counterexample $G$, i.e., $G$ is a non-solvable $\operatorname{sm}(\leq 7)$-group with $(|G|, 3)=1$. First assume that $G$ is simple. By (i) of the Lemma every maximal subgroup of $G$ is solvable and hence $G$ is isomorphic to a Suzuki group $\mathrm{Sz}(q), q=2^{2 m+1}$ (cf. [3, p. 190]). A Sylow 2-subgroup of $\mathrm{Sz}(q)$ has order $q^{2}$ and is properly contained in its normalizer. This implies that $\mathrm{Sz}(q)$ contains a proper subgroup of order $2^{n} p, p \neq 2$ a prime number, and $n \geq 6$. Therefore, $\operatorname{Sz}(q)$ possesses a maximal chain of length 8 . Consequently, containing no non-trivial submodular subgroup, $\operatorname{Sz}(q)$ cannot be an $\operatorname{sm}(\leq 7)$-group, i.e., $G$ is not simple.

The remainder of the argument runs along the lines of the proof of (i). Again, $G$ contains a unique minimal normal subgroup $N=N_{1} \times \cdots \times N_{r}$ which is non-abelian. If $P$ is a subgroup of order 2 in $N_{1}$ with $P \leq S \in \operatorname{Syl}_{2}(N)$, then $P$ is properly contained in a maximal and non-modular subgroup $V$ of $G$. Since $3 \nmid|G|$ we have $2^{6} \leq|S|$ which implies that $P$ is an $i$-maximal subgroup of $V$ for some $i \geq 6$ and hence submodular in $N_{1}$, a contradiction.

This completes the proof of the theorem.

Remarks. (i) For every natural number $i$ there exists a finite $\operatorname{sm}(\leq i)$-group with $v(G)=i+1$. Let $s=p_{1} \cdots p_{i}$ be the product of $i$ prime numbers. There exists a prime $q$ such that $q=k s+1, k \geq 1$. If $N$ is a cyclic group of order $q$, then its automorphism group $A$ is cyclic of order $q-1=k s$. Put $G=N \lambda H$ where $H$ denotes the subgroup of order $s$ in $A$. Since $G$ is supersolvable every minimal subgroup of $G$ is submodular in $G$ ([6, Lemma 6]). Hence $G$ is an $\operatorname{sm}(\leq i)$-group. It follows from $H_{G}=1$ that $v(G)=i+1$. (ii) Call a group an $\operatorname{sm}(i)$-group if each $i$-maximal subgroup is submodular. As a consequence of our theorem, if $i \leq 3$, then an $\operatorname{sm}(i)$-group is solvable. It seems unclear whether a finite $\operatorname{sm}(\leq 3)$-group is already an $\operatorname{sm}(1)-, \operatorname{sm}(2)$-, or $\operatorname{sm}(3)$-group. More generally, the following question remains open: If $G$ is an $\operatorname{sm}(\leq i)$-group, does there exist $j \leq i$ such that $G$ is an $\operatorname{sm}(j)$-group?

\section{REFERENCES}

1. M. Asaad, Generalization of a theorem of Deskins, Ann. Univ. Sci. Budapest. Eötvös Sect. Math. 18 (1975), 177-179.

2. W. E. Deskins, A condition for the solvability of a finite group, Illinois J. Math. 5 (1961), 306-313.

3. B. Huppert, Endliche Gruppen I, Springer-Verlag, Berlin-Heidelberg-New York, 1979.

4. B. Huppert and N. Blackburn, Finite groups III, Springer-Verlag, Berlin-Heidelberg-New York 1982. 
5. R. Schmidt, Modulare Untergruppen endlicher Gruppen, Illinois J. Math. 13 (1969), 358-377.

6. I. Zimmermann, Submodular subgroups in finite groups, (to appear in Math. Z).

Mathematisches Institut der Universitaet, Albertstrasse 23B, D-7800 Freiburg, West GERMANY

Current address: Department of Mathematics, Bucknell University, Lewisburg, Pennsylvania 17837, USA 